\title{
Delimitação de bacia hidrográfica em região montanhosa a partir de diferentes modelos digitais de elevação ${ }^{1}$
}

\author{
Delineation of watershed in a mountainous area using different \\ digital elevation models
}

\author{
Roberto Avelino Cecílio ${ }^{2 *}$; Luciano Melo Coutinho3\%; Alexandre Cândido Xavier ${ }^{4}$; \\ Michel Castro Moreira ${ }^{5}$; Sidney Sara Zanetti ${ }^{6}$; Giovanni de Oliveira Garcia ${ }^{7}$
}

\begin{abstract}
Resumo
A correta delimitação dos divisores de água da uma bacia hidrográfica é de grande importância para estudos ligados à modelagem hidrológica e ambiental. Tal procedimento é realizado de forma automática em aplicativos computacionais de Sistemas de Informações Geográficas, por meio de algoritmos que identificam os divisores de águas a partir de uma representação matricial da topografia do terreno, denominada Modelo Digital de Elevação (MDE). O presente trabalho avaliou a delimitação automática de uma bacia hidrográfica situada em região montanhosa do Sul do Estado do Espírito Santo (Brasil) feita a partir de seis diferentes MDEs: três MDEs originários de imagem de radar (SRTM) e seus refinamentos, além de três MDEs originários de processos de interpolação espacial de curvas de nível por meio de diferentes formas de interpolação. Verificou-se que o MDE gerado a partir das curvas de nível e da hidrografia mapeada utilizando o interpolador Topo To Raster apresentou o melhor desempenho de representação do relevo da bacia para fins de delimitação da bacia hidrográfica analisada.
\end{abstract}

Palavras-chave: Modelagem hidrológica, sistemas de informação geográfica, interpolação

\begin{abstract}
The precise delineation of watersheds is essential to studies related to environmental and hydrologic modeling. Such delineation is performed automatically in GIS softwares using algorithms that identify the watershed from grid representation of the terrain, the digital elevation model (DEM). This study evaluated the automatic delineation of a watershed located in the southern mountainous region of Espirito Santo (Brazil) using six different DEMs. Three MDEs were obtained by radar images (SRTM) and its refinements. Other three MDEs were obtained by process spatial interpolation of topographic data using different interpolators. It was found that the MDE obtained by the interpolation of topographic data using Top To Raster interpolator, (taking mapped hydrography as support) promoted the best representation of the watershed topography for the purpose of its delimitation.
\end{abstract}

Key words: Hydrologic modeling, topographic data, interpolation

\footnotetext{
${ }^{1}$ Parte da dissertação de Mestrado em Ciências Florestais do segundo autor apresentada à UFES, 2010.

${ }^{2}$ Eng $^{\circ}$ Agrícola, Prof. Dr. do Dept ${ }^{\circ}$ de Ciências Florestais e da Madeira, Universidade Federal do Espírito Santo, UFES, Alegre, ES. Bolsista de Prosutividade em Pesquisa do CNPq. E-mail: roberto.cecilio@ufes.br

${ }^{3}$ Licenciado em Geografia, Prof. M.e da Universidade São Camilo, Cachoeiro de Itapemirim, ES. E-mail: 1cncoutinho@hotmail. com

${ }^{4}$ Eng $^{\mathrm{o}}$ Agrícola, Prof. Dr. do Dept ${ }^{\circ}$ de Engenharia Rural, UFES, Alegre, ES. E-mail: alexandre.xavier@ufes.br

5 Bacharel em Ciência da Computação, Prof. Dr. do ICADS, UFBA, Barreiras, BA. E-mail: michelcm@gmail.com

${ }^{6}$ Eng $^{\circ}$ Agr $^{\circ}$, Prof. Dr. do Dept ${ }^{\circ}$ de Ciências Florestais e da Madeira, UFES. Alegre, ES. E-mail: sidney.zanetti@ufes.br

7 Eng $^{\circ}$ Agr $^{\circ}$, Prof. Dr. do Dept ${ }^{\circ}$ de Engenharia Rural, UFES. Alegre, ES. E-mail: giovanni.garcia@ufes.br

* Autor para correspondência
} 


\section{Introdução}

Entende-se por bacia hidrográfica uma unidade geográfica delimitada topograficamente por um divisor de águas, que drena as águas superficiais para uma única saída, denominada foz (CHOI; ENGEL, 2003). As bacias hidrográficas vêm se consolidando como unidades de planejamento integrado do uso e ocupação dos espaços rurais e urbanos, além da conservação dos recursos naturais, com vistas ao desenvolvimento sustentável (SOUZA; FERNANDES, 2000; VALENTE; DIAS, 2001). Dada sua importância, a Política Nacional de Recursos Hídricos (BRASIL, 1997) estabelece a bacia hidrográfica como unidade territorial de planejamento para aplicação dos instrumentos de gestão de recursos hídricos.

O aproveitamento racional dos recursos hídricos passa pelo conhecimento das disponibilidades hídricas das bacias hidrográficas, em resposta à ocupação do solo e manejo dos recursos naturais disponíveis. Neste contexto, destaca-se a importância dos modelos hidrológicos aplicados em bacias hidrográficas, a partir dos quais se podem simular diversas formas de alteração da ocupação e manejo do solo e escolher aquela cuja relação custo/benefício promova mais impactos positivos sobre a produção de água, contribuindo para a sua adequada gestão. Segundo Chang (2009), os custos associados à modelagem hidrológica consistem apenas em uma pequena fração dos custos totais de implantação de programas eficientes de manejo de bacias hidrográficas. Por outro lado, os custos associados aos resultados de um plano de manejo inadequado podem ser expressivamente maiores. Desta forma, percebe-se que uma adequada modelagem hidrológica consiste em ferramenta essencial para o manejo de bacias hidrográficas.

A delimitação correta dos divisores de água da uma bacia hidrográfica é de grande importância para estudos ligados à sua modelagem hidrológica e ambiental (RIBEIRO et al., 2008), devendo ser o primeiro passo a ser tomado na representação da área de estudo (SEYLER et al., 2009). Apenas com uma exata descrição desta unidade podemse conseguir estimativas confiáveis e consistentes de variáveis morfométricas (LEÃO et al., 2004; EHSANI; QUIEL; MALEKIAN, 2010), do comportamento hidrológico (PIRES et al., 2005; MEDEIROS; FERREIRA; FERREIRA, 2009), da qualidade da água (BAKER; WELLER; JORDAN, 2006; CHANG, 2009), da erosão hídrica (SETEGN et al., 2009), da fragilidade e dos riscos ecológicos (MANTELLI; BARBOSA; BITENCOURT, 2011) e de áreas de preservação permanente (NOWATZKI; SANTOS; PAULA, 2010).

A delimitação de bacias hidrográficas para fins de modelagem hidrológica é realizada de forma automática em aplicativos computacionais de Sistemas de Informações Geográficas. Neste processo, são utilizados algoritmos que identificam os divisores de águas a partir de uma representação matricial da topografia do terreno, denominada Modelo Digital de Elevação (MDE) (JONES; WRIGHT; MAIDMENT, 1990; FAIRFIELD; LEYMARIE, 1991; CHOI; ENGEL, 2003; ALVES SOBRINHO et al., 2010). Atualmente os MDEs são obtidos por meio de imagens de sensores remotos (radares) ou da interpolação de dados topográficos pontuais e curvas de nível extraídas de cartas topográficas ou levantamentos planialtimétricos (PINHEIRO, 2006; OLIVEIRA et al., 2010).

Os resultados da delimitação automática de uma bacia hidrográfica podem diferir significativamente dependendo das características do MDE, como escala, resolução, origem (interpolação espacial ou imagem de radar e o método de interpolação usado (REDIVO et al., 2002; ALCARAZ et al., 2009; MEDEIROS; FERREIRA; FERREIRA, 2009; SEYLER et al., 2009; LI; WONG, 2010).

Dada a importância da correta delimitação de bacias hidrográficas para a condução de estudos pertinente à modelagem hidrológica e ambiental, o presente trabalho teve como objetivo geral a avaliação da delimitação automática de uma bacia 
hidrográfica, situada em região montanhosa do Sul do Estado do Espírito Santo, por intermédio do uso de seis diferentes MDEs: três originários de imagem de radar (SRTM) e três originários de diferentes processos de interpolação espacial de curvas de nível extraídas de cartas topográficas.

\section{Material e Métodos}

A área em estudo consistiu na sub-bacia hidrográfica do rio da Prata(BHRP), inserida na bacia hidrográfica do rio Itapemirim, situada no Município de Castelo, Sul do Estado do Espírito Santo (Figura 1). Castro Júnior et al. (2007) classificaram o relevo regional pela presença de três unidades distintas: a) Faixa de Agradação Cachoeiro de Itapemirim - Castelo (altitude entre 80 e $100 \mathrm{~m}$; superfícies suaves, vales abertos e interflúvios abaulados); b) Feições de Mar de Morros (altitude entre 200 e 900 $\mathrm{m}$; solos mais resistentes ao intemperismo) e; c) Remanescentes do Ciclo Sul-Americano (altitudes em torno de $900 \mathrm{~m}$; solos espessos, com processos erosivos em franco desenvolvimento).

Figura 1. Localização da área de estudo: (a) Limites municipais do Estado do Espírito Santo, destacando o município de Castelo; e (b) Município de Castelo destacando a Bacia Hidrográfica do Rio da Prata.

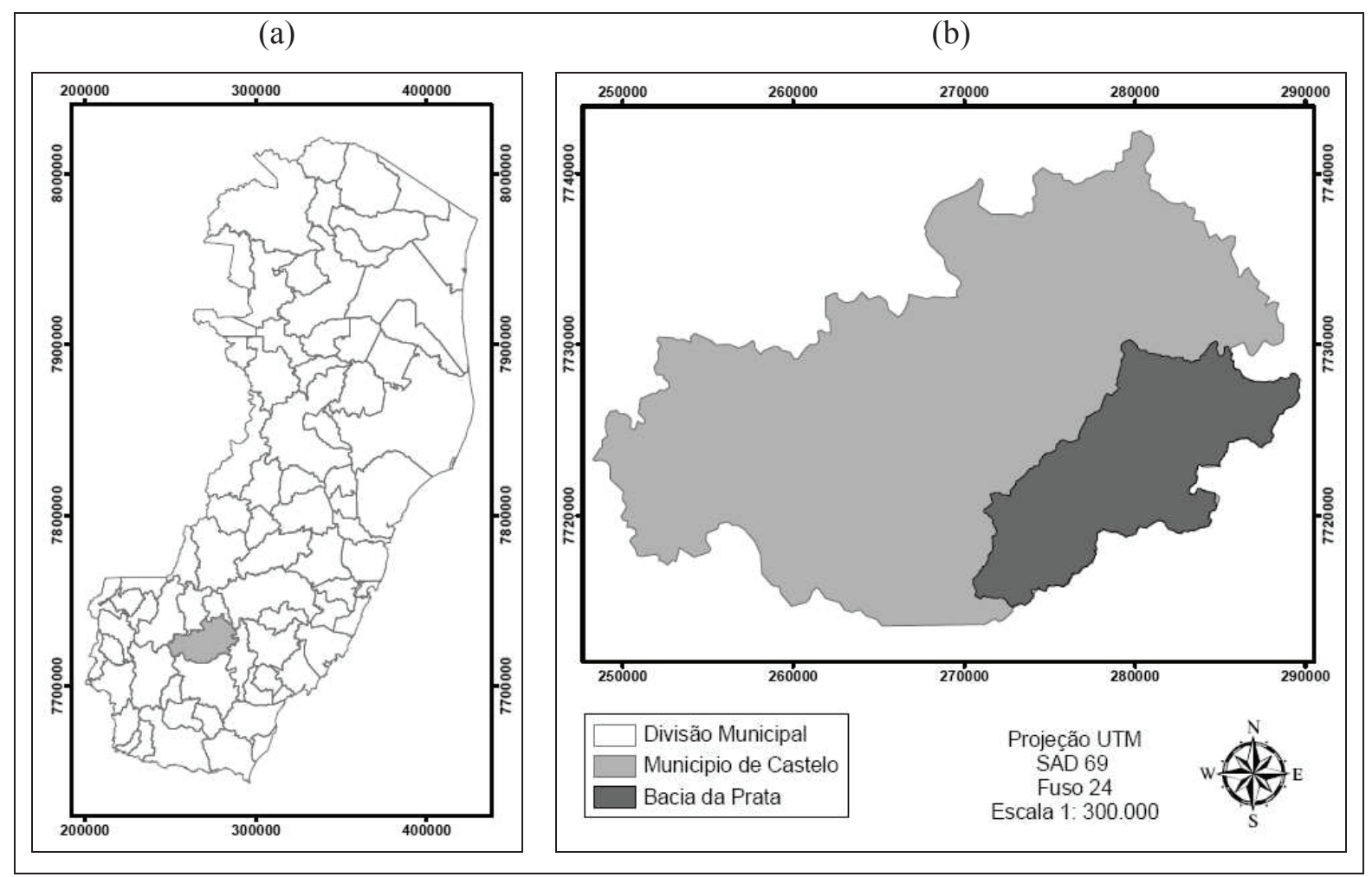

Fonte: Elaboração dos autores.

Com o intuito de avaliar as delimitações da BHRP feitas a partir de diferentes MDE's, tomou-se como referência a delimitação realizada de forma manual em carta topográfica e com suporte de aerofotos (BAKER; WELLER; JORDAN, 2006; MEDEIROS; FERREIRA; FERREIRA, 2009), doravante denominada REF. Esta delimitação foi realizada de acordo com a metodologia descrita em Cecílio et al. (2011), por meio da aplicação de técnicas de fotointerpretação para digitalização dos divisores topográficos, em computador. Para tanto, foi utilizado o aplicativo computacional ArcGIS; 
a carta topográfica na escala 1:50.000 (folha SF24-V-A-V-2) do Instituto Brasileiro de Geografia e Estatística (IBGE), contendo os dados de hidrografia e altimetria (curvas de nível com equidistância de 20 $\mathrm{m}$ ); e as aerofotos do ortofotomosaico do Espírito Santo, em escala 1:15.000, com resolução espacial de 1 x $1 \mathrm{~m}$, do Instituto Estadual de Meio Ambiente e Recursos Hídricos (IEMA).
Foram utilizados seis MDEs para a delimitação automática da bacia (Tabela 1). Três deles utilizaram a representação matricial obtida por interferometria por radar denominada SRTM (Shuttle Radar Topography Mission) (RABUS et al., 2003) e os outros três foram elaborados a partir da carta topográfica anteriormente citada.

Tabela 1. Tipos de Modelos Digitais de Elevação avaliados na delimitação da bacia hidrográfica do rio da Prata.

\begin{tabular}{cccc}
\hline Código & Base de dados original & $\begin{array}{c}\text { Interpolador/representação } \\
\text { usado }\end{array}$ & Resolução espacial \\
\hline CT-TIN & carta topográfica & TIN & $10 \mathrm{~m} \times 10 \mathrm{~m}$ \\
CT-TR & carta topográfica & TR & $10 \mathrm{~m} \times 10 \mathrm{~m}$ \\
CT-TRHD & carta topográfica & TRHD & $10 \mathrm{~m} \times 10 \mathrm{~m}$ \\
SRTM-O & imagem SRTM & - & $90 \mathrm{~m} \times 90 \mathrm{~m}$ \\
SRTM-TIN & imagem SRTM & TIN & $10 \mathrm{~m} \times 10 \mathrm{~m}$ \\
SRTM-TR & imagem SRTM & TR & $10 \mathrm{~m} \times 10 \mathrm{~m}$ \\
\hline
\end{tabular}

TIN - Triangulated irregular network; TR - Topo To Raster sem suporte de hidrografia mapeada; TRHD - Topo To Raster com suporte de hidrografia mapeada.

Fonte: Elaboração dos autores.

O MDE SRTM utilizado foi disponibilizado por Miranda (2005), com resolução espacial de 90 m x 90 m. Um MDE foi elaborado a partir da representação matricial original de SRTM; os outros dois foram realizados adaptando-se a metodologia descrita por Valeriano e Abdon (2007). Para tanto, primeiramente modificou-se o SRTM para formato vetorial (curvas de nível com equidistância de 20 $\mathrm{m})$. Para criação de um dos MDEs, as curvas de nível foram posteriormente transformadas para o formato TIN (triangulated irregular network) (JONES; WRIGHT; MAIDMENT, 1990) e convertidas para o formato matricial, gerando um MDE. Para a criação do outro MDE as curvas de nível foram interpoladas por intermédio do interpolador Topo To Raster sem suporte da hidrografia mapeada (TR) (HUTCHINSON, 1989). Desta forma, geraram-se dois MDEs SRTM refinados, ambos com resolução espacial de $10 \mathrm{~m}$ x $10 \mathrm{~m}$.

A elaboração dos três MDEs, a partir da carta topográfica, foi realizada por meio de interpolação das curvas de nível, no aplicativo computacional ArcGIS (ESRI, 2006); gerou-se dois MDEs utilizando-se dos seguintes interpoladores: Topo To Raster sem suporte da hidrografia mapeada (TR) e Topo To Raster com suporte da hidrografia mapeada (TRHD) (HUTCHINSON, 1989). No terceiro, as curvas de nível foram transformadas na representação TIN (JONES; WRIGHT; MAIDMENT, 1990) e convertidas para o formato matricial. Todas estas interpolações e conversões foram realizadas obtendo-se os MDEs com resolução espacial de 10 m x $10 \mathrm{~m}$.

Para cada MDE, a delimitação automática da bacia hidrográfica foi realizada no ArcGIS (ESRI, 2006), utilizando-se as extensões Spatial Analyst e Hydrology Modeling. Para tanto, primeiramente foram identificadas e eliminadas as depressões espúrias (células cercadas por outras com valores de elevação maiores) existentes nos MDEs, uma vez que estas podem causar distorções na rede de drenagem modelada e alterar, ou até mesmo 
impossibilitar, o traçado dos divisores de água (WISE, 2000). Tal procedimento foi feito por meio do comando Fill Sinks. Posteriormente foram definidas as direções de fluxo (comando Flow Direction), o fluxo acumulado (comando Flow Accumulation) e, finalmente, a delimitação automática da bacia (comando Watershed) (MEDEIROS; FERREIRA; FERREIRA, 2009).

A avaliação comparativa das delimitações da bacia foi realizada a partir dos seguintes critérios, indicados por Cuartero, Felicisimo e Ariza (2001), Redivo et al. (2002), Pires et al. (2005), Pinheiro (2006) e Lindsay, Rothwell e Davies (2008): a) valores de altitude máxima e mínima e da área de drenagem da bacia hidrográfica delimitada; b) localização da foz da bacia delimitada; e c) comparação visual entre os divisores de água.

\section{Resultados e Discussão}

Análise dos valores de altitude máxima e mínima e da área de drenagem das delimitações da bacia hidrográfica

A Tabela 2 apresenta os valores de área de drenagem, altitude máxima e altitude mínima obtidos nas delimitações da BHRP, por intermédio da delimitação de referência (REF) e utilizando os diferentes MDE's avaliados.

Os valores de área de drenagem indicam que aquela mais próxima à calculada a partir da delimitação tomada como padrão (REF) foi obtida usando o MDE gerado com o interpolador TRHD aplicado às curvas de nível da carta topográfica (CT-TRHD), com diferença percentual entre áreas igual a $0,04 \%$. Observa-se que o uso do TIN (CTTIN e SRTM-TIN) propiciou os maiores desvios relativos à área de drenagem, considerando-se separadamente as duas categorias de interpolação - curvas de nível (CT-TIN, CT-TR, CT-TRHD) e refinamento do SRTM (SRTM-TIN e SRTM-TR). Cecílio et al. (2011) também verificaram o mesmo comportamento de um MDE obtido com uso do TIN para a delimitação de uma bacia hidrográfica próxima à BHRP. As delimitações realizadas a partir do SRTM e seus refinamentos (SRTM-O, SRTM-TIN e SRTM-TR) apresentaram valores de área de drenagem mais discrepantes, sendo que, destas, a delimitação SRTM-TR apresentou área de drenagem mais próxima ao valor relativo à delimitação REF. Analisando-se a delimitação a partir do SRTM original (SRTM-O), Oliveira et al. (2010) encontrou diferença percentual entre áreas muito semelhante à calculada no presente trabalho, considerando-a como satisfatória.

Tabela 2. Área de drenagem e altitudes (máxima e mínima) da bacia hidrográfica do rio da Prata delimitada de forma manuale por meio de diferentes modelos digitais de elevação.

\begin{tabular}{ccccc}
\hline Código & $\begin{array}{c}\text { Área } \\
\left(\mathbf{k m}^{\mathbf{2}}\right)\end{array}$ & $\begin{array}{c}\text { Diferença entre } \\
\text { áreas } \mathbf{( \% )}\end{array}$ & $\begin{array}{c}\text { Altitude máxima } \\
\mathbf{( m )}\end{array}$ & $\begin{array}{c}\text { Altitude mínima } \\
\mathbf{( m )}\end{array}$ \\
\hline REF & 132,28 & - & entre 2020 e 2040 & entre 60 e 80 \\
CT-TIN & 133,64 & 1,02 & 2020 & 80 \\
CT-TR & 132,39 & 0,08 & 2031 & 70 \\
CT-TRHD & 132,33 & 0,04 & 2031 & 82 \\
SRTM-O & 134,66 & 1,80 & 1977 & 100 \\
SRTM-TIN & 137,54 & 3,98 & 1980 & 100 \\
SRTM-TR & 131,57 & $-0,54$ & 1986 & 72 \\
\hline
\end{tabular}

Fonte: Elaboração dos autores. 
Todos os valores de diferenças percentuais entre áreas podem ser considerados baixos quando comparados com outros estudos, como Leão et al. (2004) e Seyler et al. (2009). Estes encontraram valores variando de $1,62 \%$ e $17,41 \%$ em bacias hidrográficas no Norte e Nordeste do Brasil, situadas em regiões mais planas que a do presente estudo. Tal fato sugere o melhor desempenho de delimitação para áreas montanhosas.

Pela Tabela 2, verifica-se ainda que as delimitações CT-TR e CT-TRHD apresentaram altitude máxima (2031 m) compatível com D1, o que se justifica pelas tentativas de ajuste do relevo pelo interpolador TR para compensação da continuidade da elevação (ESRI, 2006). Este processo não é verificado no TIN, pois este apenas interliga as curvas, e determina valores de distância entre estas, desconsiderando a ocorrência de topos e vales (CÂMARA; DAVIS; MONTEIRO, 2001; NAMIKAWA et al., 2003). Os MDEs originários dos dados SRTM (SRTM-O, SRTM-TIN e SRTMTR) apresentam altitudes máximas inferiores a REF.

Considera-se importante a observação dos valores de altitude mínima, pois se referem à foz da bacia. Os MDEs originários das curvas de nível do IBGE (CT-TIN, CT-TR e CT-TRHD) apresentaram as menores discrepâncias de altitude mínima. Isto se explica pelo fato de que estes MDEs são derivados da interpolação das próprias curvas de nível do IBGE.

Os MDEs SRTM-O, SRTM-TIN e SRTMTR apresentaram maiores diferenças de altitude mínima, sendo $100 \mathrm{~m}$ para SRTM-O e SRTM-TIN, em função de tamanho do pixel (90 m) e do modelo de triangulação dos valores de altitude das isolinhas, respectivamente, considerados os piores resultados. O pior desempenho altimétrico do MDE-SRTM e seus refinamentos em muito se deve à sua precisão vertical, que, em média, é de $16 \mathrm{~m}$, com 90\% de exatidão (RABUS et al., 2003); contudo, cabe ressaltar que podem sofrer influência da vegetação, que pode elevar o erro a até 25 metros, conforme evidenciado por Miliaresis e Paraschou (2005); Ludwig e Schneider (2006); Santos, Gaboardi e Oliveira (2006); Valeriano e Abdon (2007) e Costa et al. (2010). Além disso, de acordo com Moreira (2005), os dados de radar são oriundos de sensores ativos que se pautam no envio e retorno de sinal, cuja intensidade é proporcional ao nível de cinza registrado. O sinal de retorno está sujeito a respostas distintas devido a sua interação com os diferentes tipos de superfície (relevo, vegetação, hidrografia), o que pode omitir ou exagerar a representação do relevo, apresentando algumas imperfeições como valores extremamente altos e baixos (picos e vórtices), linhas de costa e corpos d'água mal definidos (RABUS et al., 2003; PINHEIRO, 2006).

\section{Localização da foz da bacia delimitada}

$\mathrm{Na}$ Figura 2 são apresentadas as regiões de localização da foz da BHRP delimitadas com o uso dos diferentes MDEs. Verifica-se que o método automático gerou erros de delimitação de áreas de contribuição direta para o canal principal e deslocamentos da foz em todos MDEs. Os menores erros na localização da foz da bacia se deram nas delimitações CT-TRHD, CT-TR e CTTIN (MDEs gerados a partir da carta topográfica), com deslocamentos iguais a 15, 71 e 118 metros, respectivamente. As delimitações geradas a partir do SRTM, isto é, SRTM-O, SRTM-TIN e SRTM-TR, apresentaram os erros maiores, com deslocamentos iguais a 614, 1.816 e 421 metros, respectivamente. Tal fato se deveu à descaracterização da rede hidrográfica por meio da simulação de cursos d'água retilíneos na área mais plana. Seyler et al. (2009) considera este como um problema inerente à identificação de redes de drenagem numérica em áreas planas. Fairfield e Leymarie (1991) e Pires et al. (2005) sugerem que a rede de drenagem mapeada seja usada para forçar a localização da drenagem. Este procedimento é feito ao se utilizar o interpolador Topo To Raster com suporte da hidrografia (CT-TRHD), conforme realizado no presente trabalho. 
Figura 2. Comparação visual da localização da foz da bacia hidrográfica do rio da Prata obtida pela delimitação de referência (REF) e pelas delimitações automáticas.

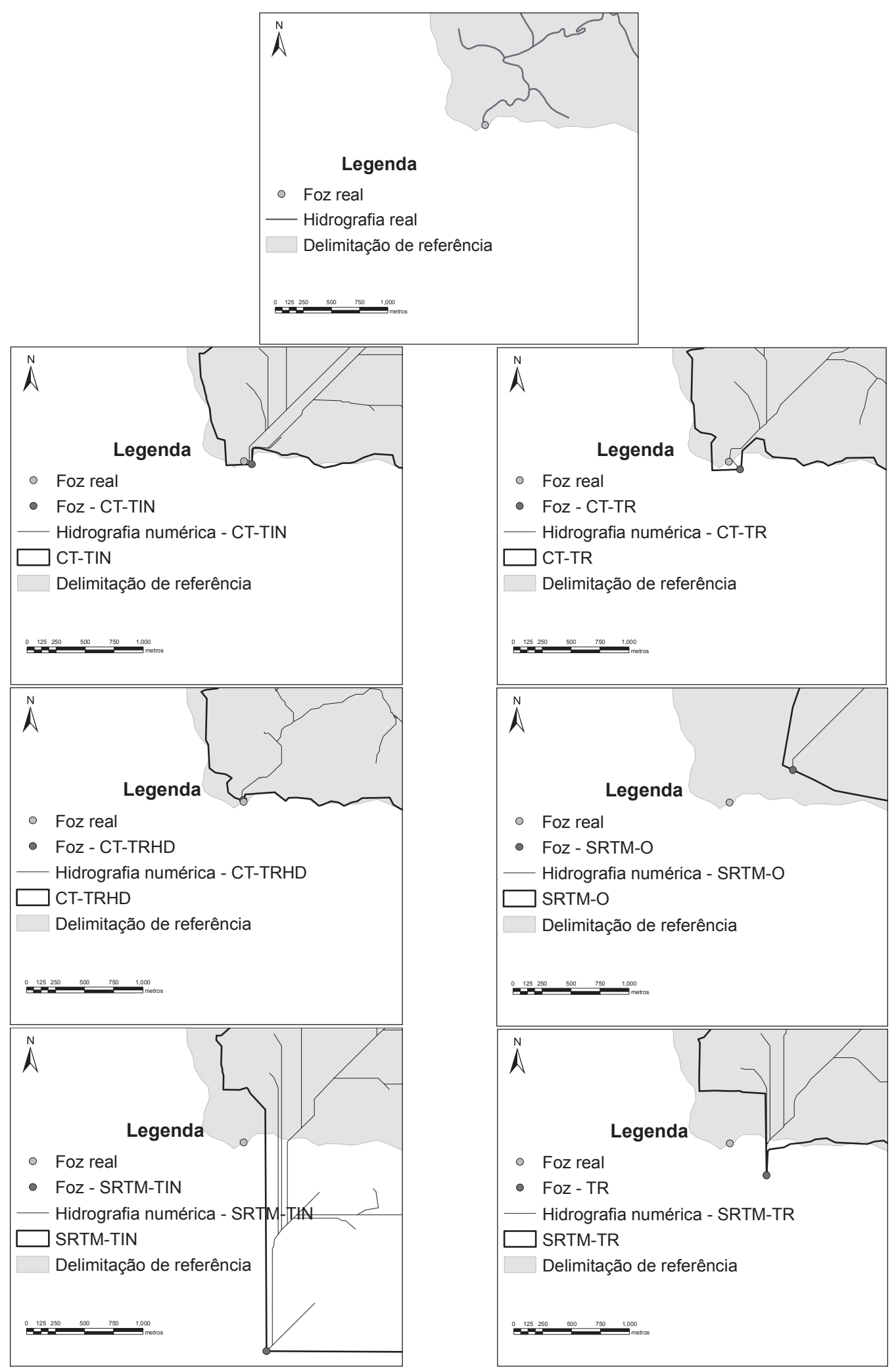

Fonte: Elaboração dos autores. 
Comparação visual entre os divisores de água

A Figura 3 apresenta os erros mais nítidos verificados na comparação entre REF e CT-TIN. Observam-se erros de exclusão de áreas contendo rede hidrográfica (Figura 3A e Figura 3D); inclusão de área e de parte da hidrografia da bacia vizinha (Figura 3B); e sequências de pequenos erros de inclusão ou de exclusão de áreas (Figura 3C e Figura 3E). Tais erros podem estar associados ao princípio da representação TIN, uma vez que a ligação direta entre as curvas de nível pode desconsiderar vales estreitos, mesmo com a adoção de pixels de menor resolução. Utilizando MDE obtido com o TIN, Alcaraz et al. (2009) e Cecílio et al. (2011) também verificaram os mesmos tipos de erros na delimitação de bacias hidrográficas no Brasil. Segundo Alcaraz et al. (2009), o MDE advindo do TIN caracteriza-se por uma superfície irregular composta por sucessivos degraus planos, não representando satisfatoriamente uma superfície topográfica de variação contínua e, por isso, possui pior desempenho.

Os erros verificados entre as delimitações REF e CT-TR são apresentados na Figura 4. Nota-se menor número de erros, comparativamente a CTTIN, com poucos cortes ou inclusões de áreas e de cursos da hidrografia, sendo os erros mais nítidos: inclusão de área (Figura 4A); exclusão de área e passagem do divisor sobre um canal (Figura 4B); e sequência de inclusão de canal e de área de bacia vizinha e uma exclusão de área (Figura 4C). Assim, utilizando as curvas de nível do IBGE, o MDE advindo do interpolador TR, mesmo sem o suporte da hidrografia mapeada, apresentou melhor desempenho que o MDE obtido usando o TIN.

$\mathrm{Na}$ Figura 5 constam as diferenças verificadas entre REF e CT-TRHD (delimitação a partir do MDE gerado por meio da interpolação de curvas de nível por intermédio do TRHD). A delimitação
CT-TRHD foi a que apresentou os menores erros em relação à delimitação de referência, com base na comparação de dados de mapeamento, sendo estes: pequena inclusão de área (Figura 5A) e pequenas exclusões de áreas (Figuras 5B e 5C). Este MDE havia apresentado o menor erro de área de drenagem em relação à delimitação manual (Tabela 2), e mostrou um grande diferencial por não traçar o divisor topográfico sobre a rede hidrográfica da BHRP e por não incluir canais de bacias vizinhas. Pires et al. (2005), Alcaraz et al. (2009) e Medeiros, Ferreira e Ferreira (2009) também classificaram este como o melhor interpolador, dentre os avaliados, para gerar MDEs com fins de delimitação de bacias hidrográficas, corroborando o resultado aqui encontrado. Todavia, Medeiros, Ferreira e Ferreira (2009) encontraram melhores resultados quando da utilização do MDE-SRTM (equivalente a SRTM-O no presente estudo).

A Figura 6 apresenta os erros mais nítidos verificados na comparação entre REF e SRTM-O. Os principais erros verificados foram: inclusão de área e canal de bacia vizinha (Figura 6A e 6B); sequência de erros de inclusões de áreas e canais das bacias vizinhas (Figura 6C e 6D); e exclusão de área de bacia vizinha próxima a foz (Figura 6E). Medeiros, Ferreira e Ferreira (2009) encontraram o SRTM original como o melhor MDE para delimitação de grandes bacias hidrográficas em Goiás. Todavia, a escala de trabalho era maior, o que reduz a influência do tamanho do pixel; além disso, os próprios autores relataram que a precisão dos dados SRTM é altamente correlacionada à declividade, sendo menor a precisão em áreas com maior declividade. Desta forma, justifica-se o pior desempenho de SRTM-O na delimitação da BHRP, pois esta se insere em uma região montanhosa, caracterizada por altas declividades. O SRTM pode propocionar melhores resultados de delimitação por meio de seu refinamento utilizando um interpolador adequado. 
Figura 3. Comparação entre a delimitação de referência (REF) e a delimitação automática a partir da interpolação das curvas de nível usando TIN (CT-TIN).
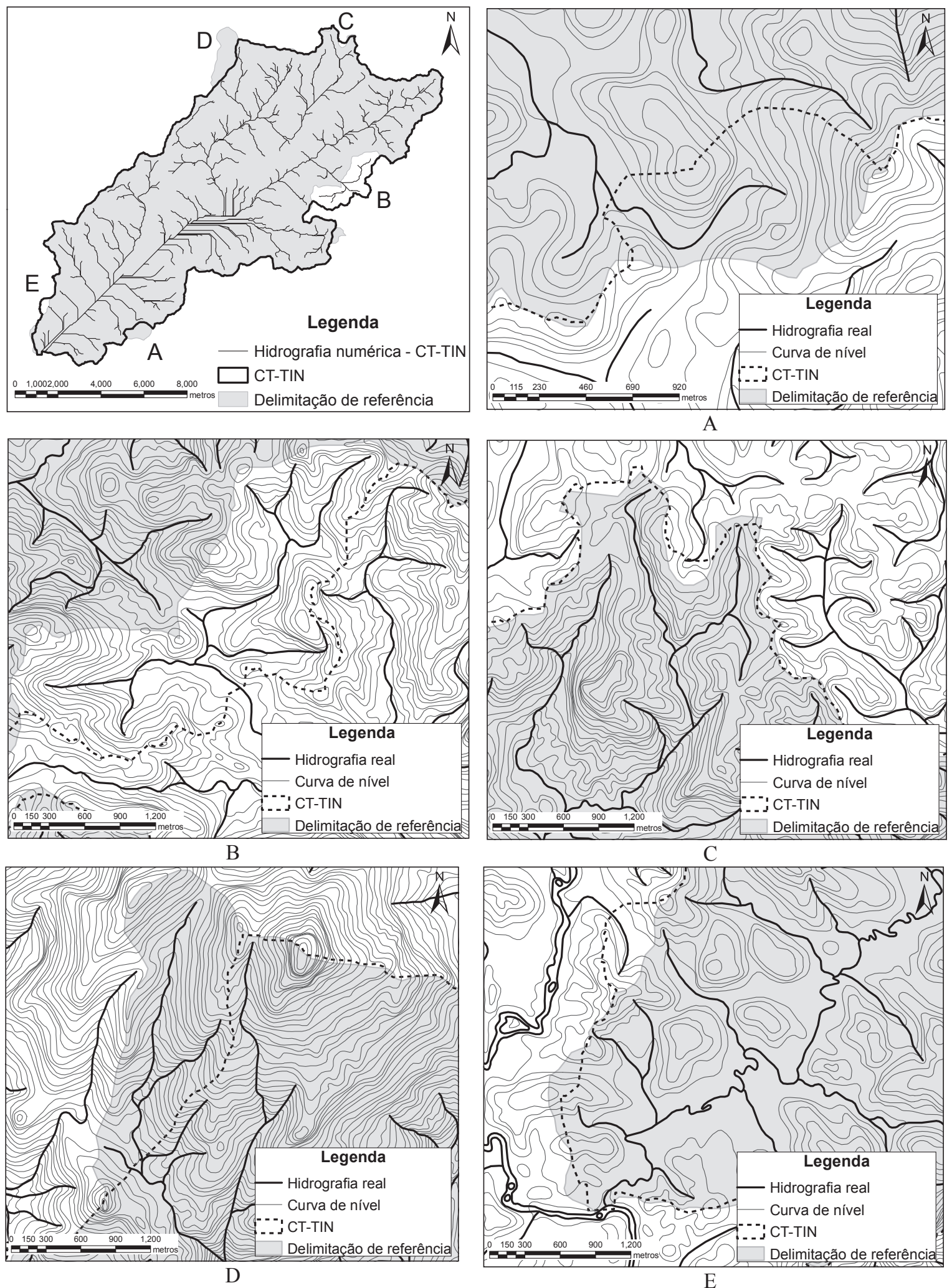

Fonte: Elaboração dos autores. 
Figura 4. Comparação entre a delimitação de referência (REF) e a delimitação automática a partir da interpolação das curvas de nível usando Topo To Raster (CT-TR).
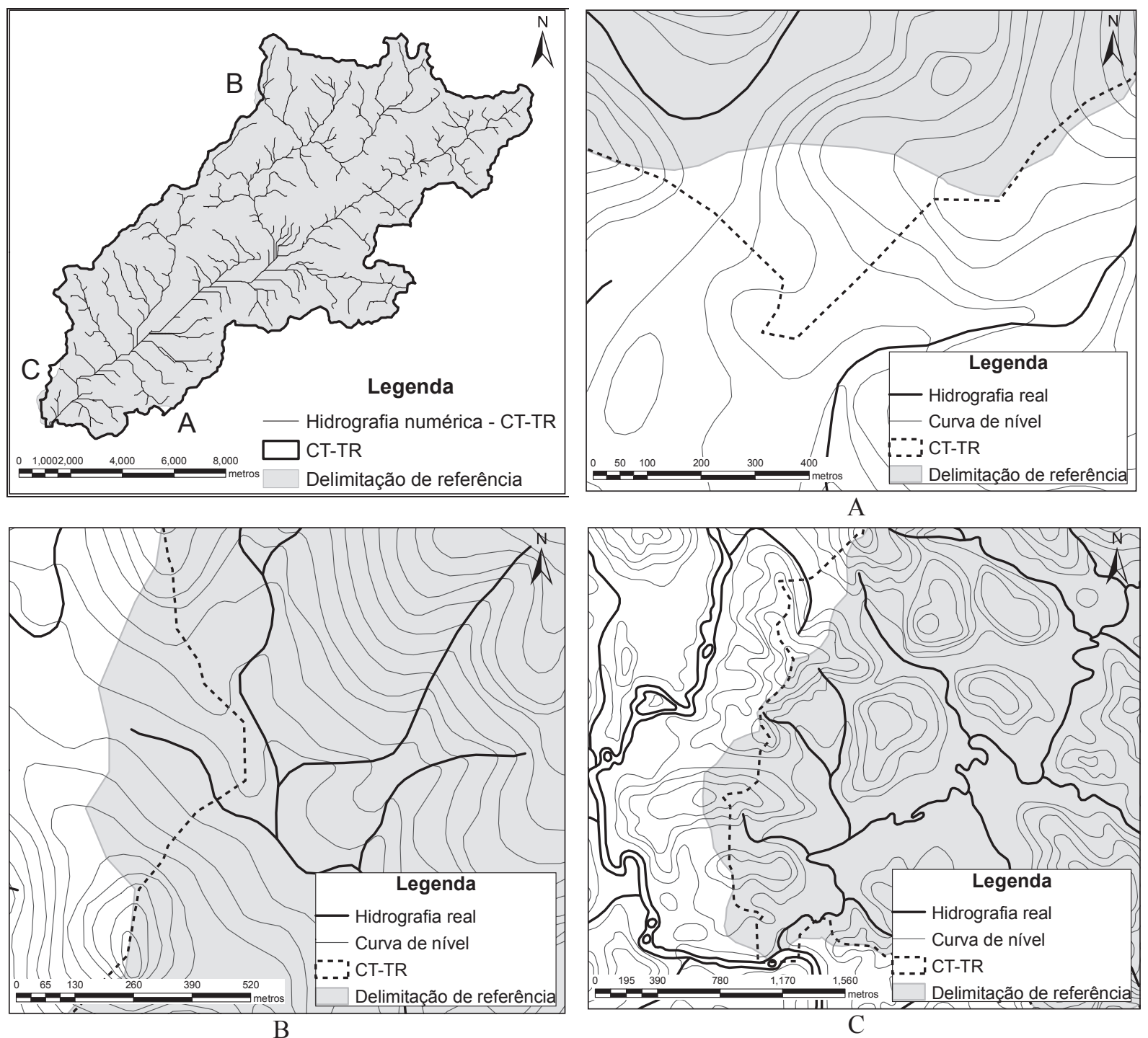

Fonte: Elaboração dos autores. 
Figura 5. Comparação entre a delimitação de referência (REF) e a delimitação automática a partir da interpolação das curvas de nível usando Topo To Raster com suporte da hidrografia (CT-TRHD).

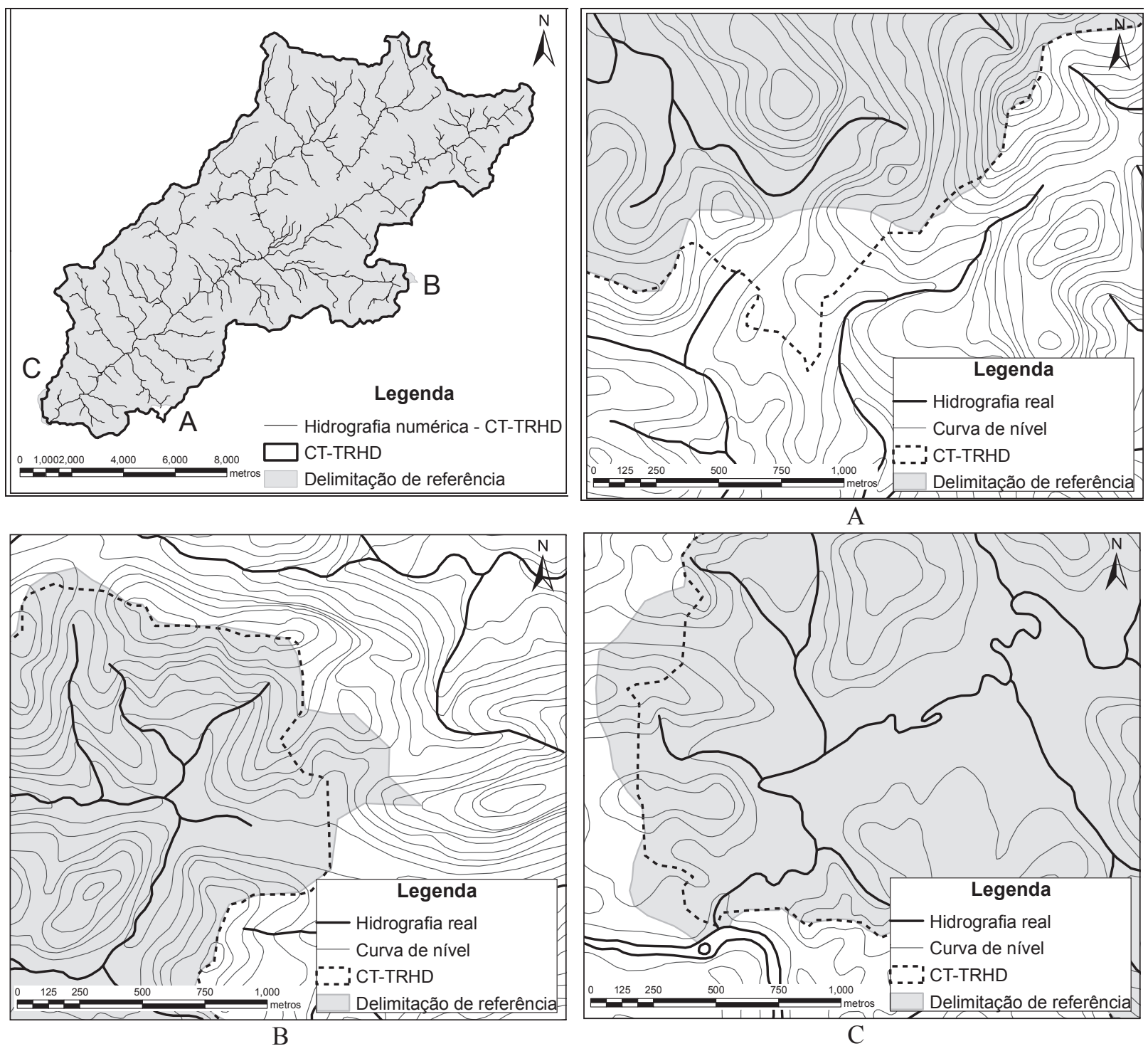

Fonte: Elaboração dos autores. 
Figura 6. Comparação entre a delimitação de referência (REF) e a delimitação automática a partir do MDE-SRTM original (SRTM-O).
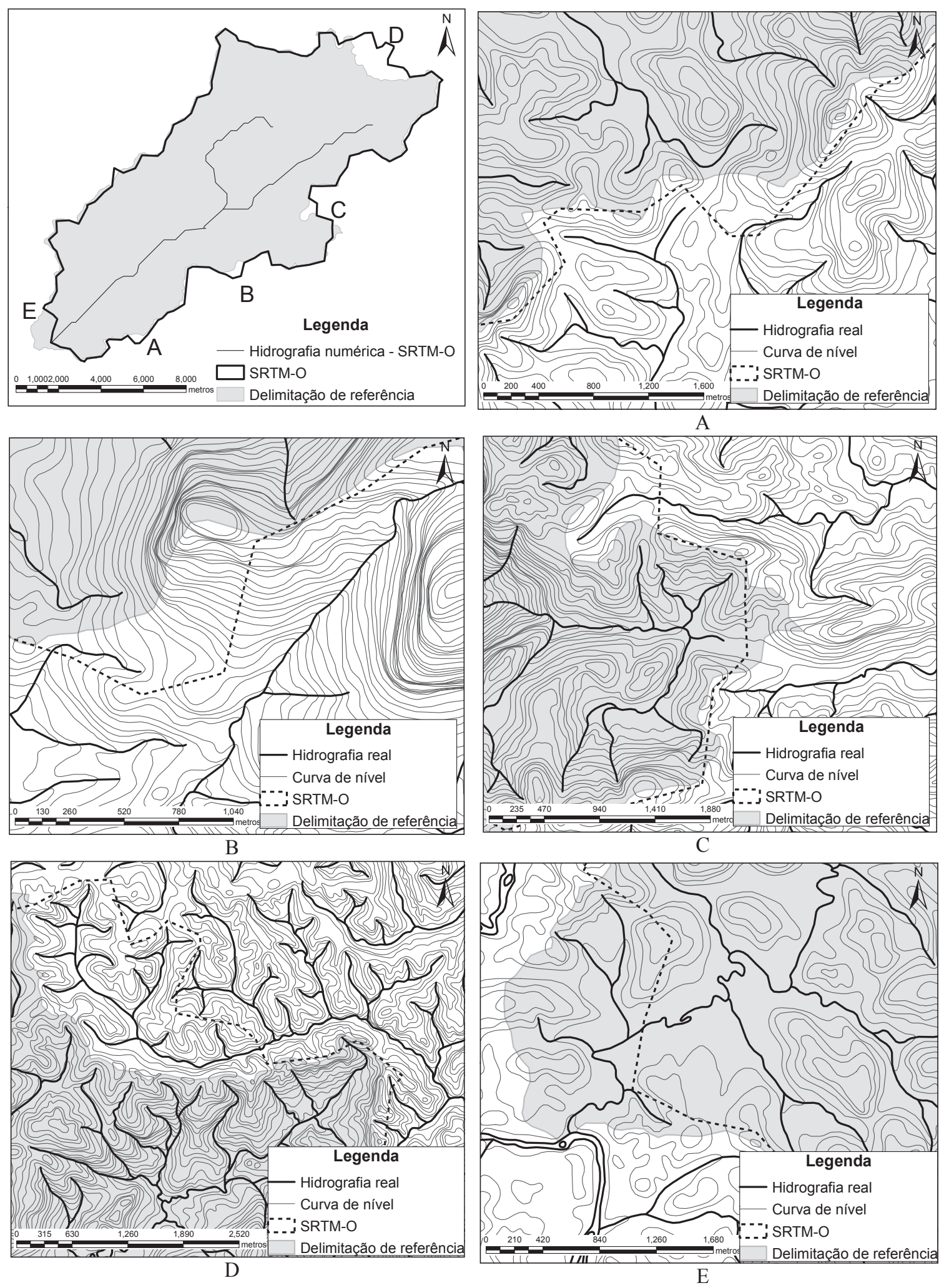

Fonte: Elaboração dos autores. 
Os erros verificados entre a delimitação REF e SRTM-TIN são ilustrados na Figura 7. Observamse os seguintes erros: inclusão de áreas e canais de bacias vizinhas (Figura 7Ae 7C); e exclusões de áreas (Figura 7B, 7D e 7E). Verifica-se que o refinamento do MDE SRTM por meio do TIN resultou em pior delimitação da bacia hidrográfica, indicando que o TIN não é adequado para refinamento dos dados SRTM, corroborando as conclusões obtidas por Kumler (1994) ao estudar uma série de bacias hidrográficas nos Estados Unidos.

Figura 7. Comparação entre a delimitação de referência (REF) e a delimitação automática usando o MDE-SRTM refinado por meio do TIN (SRTM-TIN).
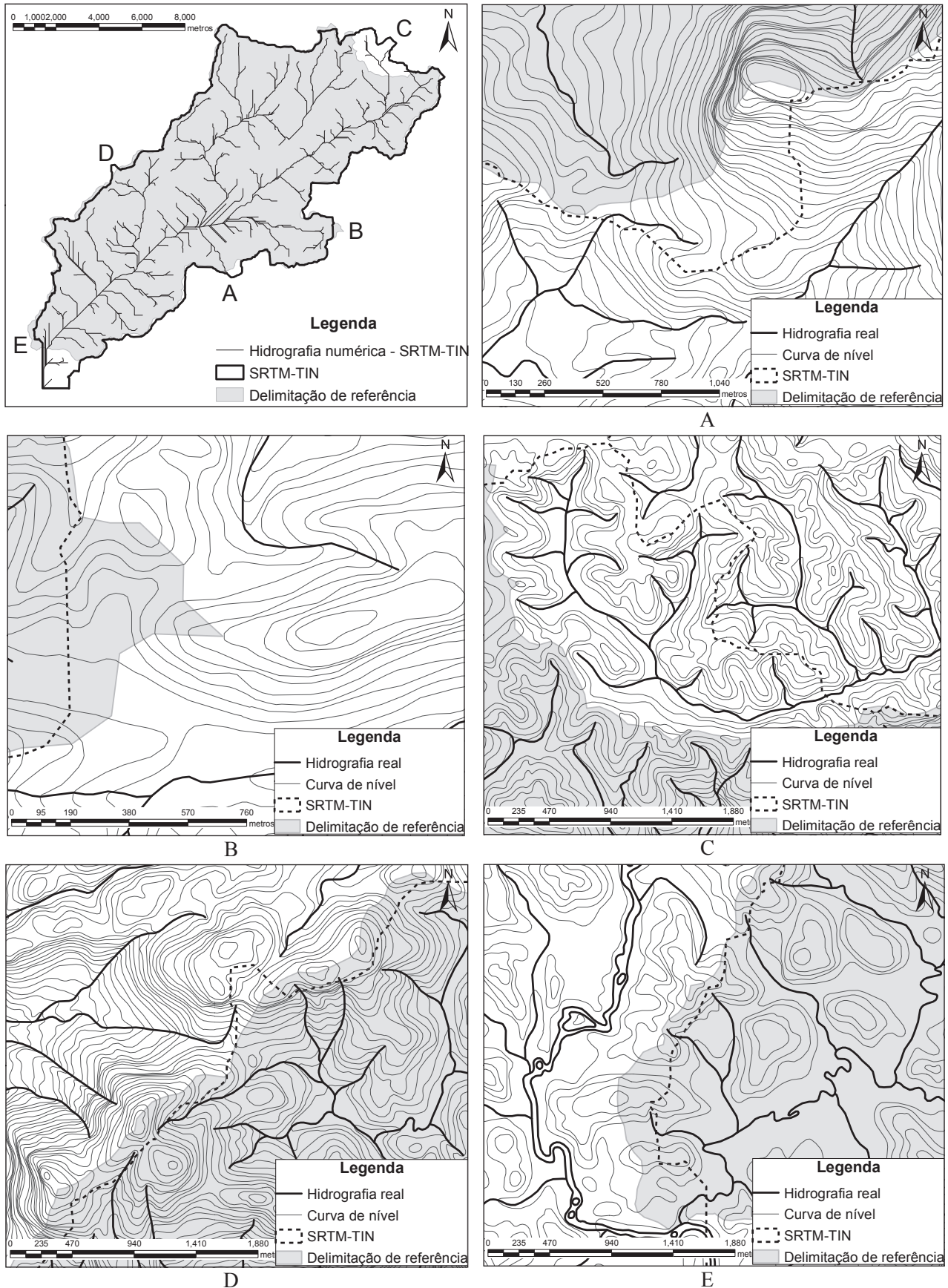

Fonte: Elaboração dos autores. 
Os erros verificados entre as delimitações REF e SRTM-TR são ilustrados na Figura 8. As principais observações são: erros de inclusão de área (Figura 8A); inclusão de área e canal da bacia vizinha (Figura 8B); inclusões e exclusões de áreas e canais (Figura 8C e 8E); e erros de exclusão de área (Figura 8D). Esta delimitação apresentou menores erros devido ao uso de dados
SRTM refinados, o que mostra a importância em selecionar o interpolador mais adequado. Embora não avaliados no presente trabalho, outras publicações mostraram bons desempenhos de outros interpoladores no refinamento do STRM, como a Convolução Cúbica, Bicúbica e Krigagem (EHSANI; QUIEL; MALEKIAN, 2010; METZ; MITASOVA; HARMON, 2010; VALERIANO; ROSSETTI, 2012).

Figura 8. Comparação entre a delimitação de referência (REF) e a delimitação automática a partir do MDE-SRTM refinado usando Topo To Raster sem suporte da hidrografia (SRTM-TR).
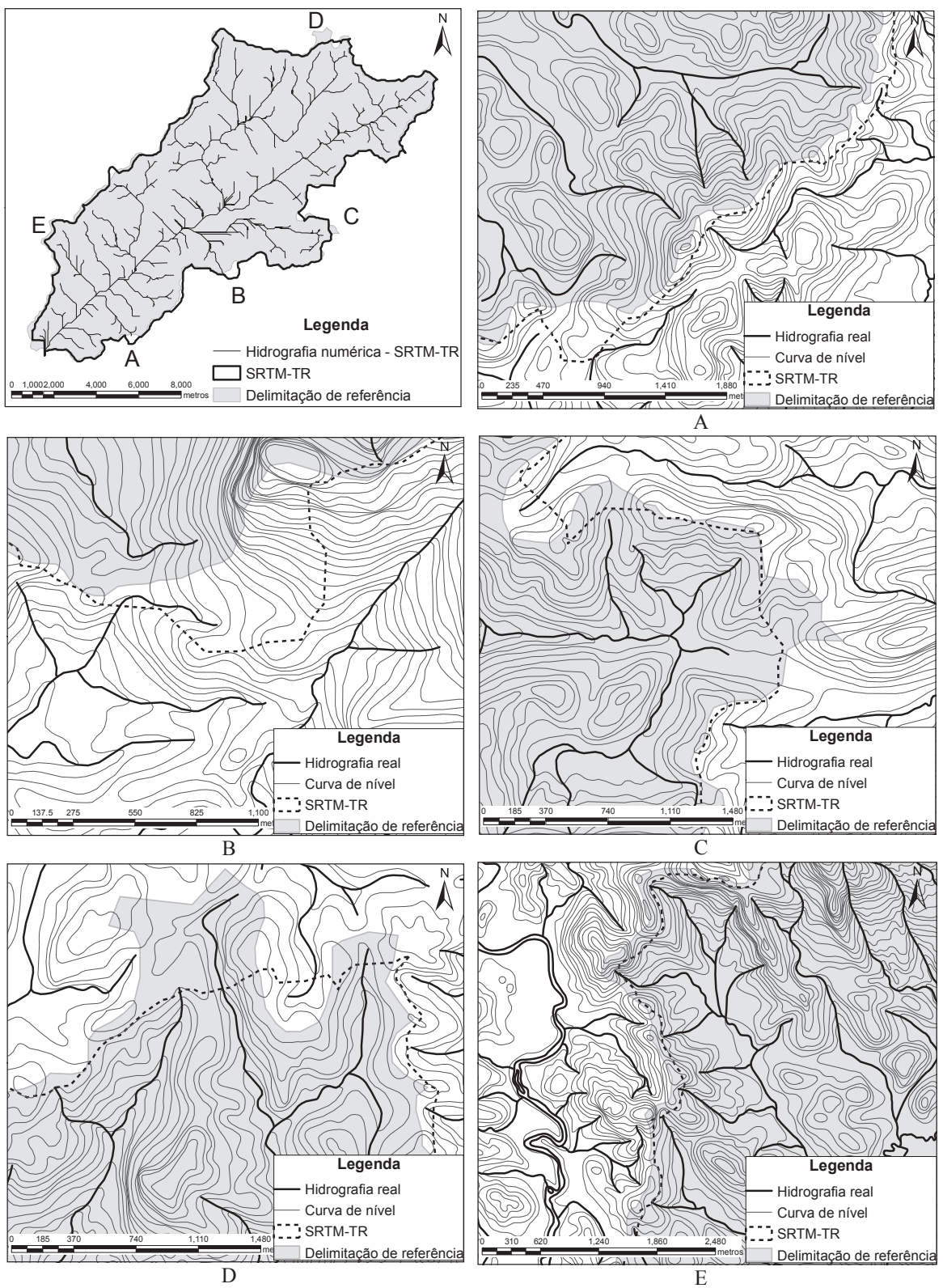

Fonte: Elaboração dos autores. 
Com base na comparação visual dos procedimentos de delimitação, os menores erros gerais foram verificados na delimitação CTTRHD, utilizando-se o MDE gerado por meio da interpolação de curvas de nível com o interpolador Topo To Raster com suporte de hidrografia mapeada (TRHD).

Com base nos resultados apresentados pode-se concluir que:

1. O modelo digital de elevação originário da interpolação das curvas de nível da carta topográfica do IBGE por intermédio do interpolador Topo To Raster, com suporte da hidrografia mapeada apresentou o melhor desempenho na delimitação da bacia hidrográfica do rio da Prata.

2. A utilização do modelo digital de elevação originário do refinamento do SRTM usando o interpolador Topo To Raster apresentou resultados satisfatórios na delimitação da bacia hidrográfica do rio da Prata.

3. O uso do TIN para criação ou refinamento do modelo digital de elevação apresentou os piores resultados na delimitação da bacia hidrográfica do rio da Prata.

\section{Referências}

ALCARAZ, S. A.; SANNIER, C.; VITORINO, A. C. T.; DANIEL, O. Comparison of methodologies for automatic generation of limits and drainage networks for hidrographic basins. Revista Brasileira de Engenharia Agrícola e Ambiental, Campina Grande, v. 13, n. 4, p. 369-375, 2009.

ALVES SOBRINHO, T.; OLIVEIRA, P. T. S.; RODRIGUES, D. B. B.; AYRES, F. M. Delimitação automática de bacias hidrográficas utilizando dados SRTM. Engenharia Agrícola, Jaboticabal, v. 30, n. 1, p. 46-57, 2010.

BAKER, M. E.; WELLER, D. E.; JORDAN, T. E. Comparison of automated watershed delineations: effects on land cover areas, percentages, and relationships to nutrient discharge. Photogrammetric Engineering \& Remote Sensing, Bethesda, v. 72, n. 2, p. 159-168, 2006.
BRASIL, Política Nacional de Recursos Hídricos. Lei no 9.433, de 8 de janeiro de 1997. [S.1.]: MMA/SRH, 1997. CÂMARA, G.; DAVIS, C.; MONTEIRO, A. M. V. Introdução à ciência da geoinformação. INPE: São José dos Campos, 2001. 345 p.

CASTRO JÚNIOR, R. M.; SOBREIRA, F. G.; BORTOLOTI, F. D.; SOUZA, L. A. Definição de unidades geomorfológicas a partir de navegação e validação de campo utilizando GPS e sistemas de informações geográficas: o caso as sub-bacia do rio Castelo-ES. Boletim de Ciências Geodésicas, v. 13, n. 1, p. 42-59, 2007.

CECÍLIO, R. A.; COUTINHO, L. M.; ZANETTI, S. S.; GARCIA, G. O. Delimitação de bacias hidrográficas. In: ANDRADE, F. V.; PASSOS, R. R.; MENDONÇA, E. S.; LIMA, J. S. S.; FERREIRA, A. (Org.). Tópicos especiais em produção vegetal II. Alegre: Centro de Ciências Agrárias, 2011. p. 329-348.

CHANG, C. L. The impact of watershed delineation on hydrology and water quality simulation. Environmental Monitoring and Assessment, v. 148, n. 1-4, p. 159-165, 2009.

CHOI, J. Y.; ENGEL, B. A. Real-time watershed delineation system using web-GIS. Journal of Computing in Civil Engineering, v. 17, n. 3, p. 189-196, 2003.

COSTA, C. A. G.; TEIXEIRA, A. S.; ANDRADE, E. M.; LUCENA, A. M. P.; CASTRO, M. A. H. Análise da influência vegetacional na altimetria dos dados SRTM em bacias hidrográficas no semiárido. Revista Ciência Agronômica, Fortaleza, v. 41, n. 2, p. 222-230, 2010.

CUARTERO, A.; FELICISIMO, A. M.; ARIZA, F. J. Análises del error em modelos digitales de elevaciones según la fuente de dados. In: CONCRESSO INTERNACIONAL DE ESPRESIÓN GRÁFICA, 12., 2001, Badajoz. Anais... Badajoz: [s.n], 2001. Disponível em:<http://www.ingegraf.es/congresos/2001BADAJOZ/docs/730656.doc >. Acesso em: 26 out. 2010.

EHSANI, A. H.; QUIEL, F.; MALEKIAN, A. Effect of SRTM resolution on morphometric feature identification using neural network-self organizing map. Geoinformatica, v. 14, n. 4, p. 405-424, 2010.

ENVIRONMENTAL SYSTEMS RESEARCH INSTITUTE - ESRI. ArcGIS Professional GIS for the desktop, version 9.2. Software. 2006.

FAIRFIELD, J.; LEYMARIE, P. Drainage networks from grid digital elevation models. Water Resources Research, v. 30, n. 6, p. 1681-1692, 1991. 
HUTCHINSON, M. F. A new procedure for gridding elevation and stream line data with automatic removal of spurious pits. Journal of Hydrology, Amsterdan, v. 106, n. 3, p. 211-232, 1989.

JONES, N. L.; WRIGHT, S. G.; MAIDMENT, D. R. Watershed delineation with triangle-based terrain models. Journal of Hydraulic Engineering, v. 116, n. 10, p. 1232-1252, 1990.

KUMLER, M. P. An intensive comparison of triangulated irregular networks (TINs) and digital elevation models (DEMs). Cartographica, v. 31, n. 2, p. 1-99, 1994.

LEÃO, R. A. O.; TEIXEIRA, A. S.; ANDRADE, E. M.; OLIVEIRA, F. Delimitação e caracterização automática de uma micro-bacia hidrográfica da Fazenda Experimental Vale Curu, Pentecoste - CE. Revista Ciência Agronômica, Fortaleza, v. 35, n. 1, p. 26-35, 2004.

LI, J.; WONG, D. W. S. Effects of DEM sources on hydrologic applications. Computers, Environment and Urban Systems, v. 34, n. 3, p. 251-261, 2010.

LINDSAY, J. B.; ROTHWELL, J. J.; DAVIES, H. Mapping outlet points used for watershed delineation onto DEM derived stream networks. Water Resources Research, v. 44, p. W08442, 2008.

LUDWIG, R.; SCHNEIDER, P. Validation of digital elevation models from SRTM X-SAR for applications in hydrologic modeling. ISPRS Journal of Photogrammetry \& Remote Sensing, v. 60, n. 5, p. 339-358, 2006.

MANTELLI, L. R.; BARBOSA, J. M.; BITENCOURT, M. D. Assessing ecological risk through automated drainage extraction and watershed delineation. Ecological Informatics, v. 6, n. 5, p. 325-331, 2011.

MEDEIROS, L. C.; FERREIRA, N. C.; FERREIRA, L. G. Avaliação de modelos digitais de elevação para delimitação automática de bacias hidrográficas. Revista Brasileira de Cartografia, v. 61, n. 2, p. 137-151, 2009.

METZ, M.; MITASOVA, H.; HARMON, R. S. Accurate stream extraction from large, radar-based elevation models. Hydrology Earth Systems Science Discussions, v. 7, n. 3, p. 3213-3235, 2010.

MILIARESIS, G. C.; PARASCHOU, C. V. E. Vertical accuracy of the SRTM DTED level 1 of Crete. International Journal of Applied Earth Observation and Geoinformation, v. 7, n. 1, p. 49-59, 2005.

MIRANDA, E. E. (Coord.). Brasil em relevo. Campinas: Embrapa Monitoramento por Satélite, 2005. Disponível em: <http://www.relevobr.cnpm.embrapa.br>. Acesso em: 26 out. 2010.
MOREIRA, M. A. Fundamentos do sensoriamento remoto e metodologias de aplicação. 3. ed. Viçosa: Editora UFV, 2005. 320 p.

NAMIKAWA, L. M.; FELGUEIRAS, C. A.; MURA, J. C.; ROSIM, S.; LPES, E. S. S. Modelagem numérica de terreno e aplicações. INPE: São José dos Campos, 2003. $142 \mathrm{p}$.

NOWATZKI, A.; SANTOS, L. J. C.; PAULA, E. V. Utilização do SIG na delimitação das áreas de preservação permanente (APP's) na bacia do rio Sagrado (Morretes/ PR). Sociedade \& Natureza, v. 22, n. 1, p. 107-120, 2010.

OLIVEIRA, P. T. S.; ALVES SOBRINHO, T.; STEFFEN, J. L.; RODRIGUES, D. B. B. Caracterização morfométrica de bacias hidrográficas através de dados SRTM. Revista Brasileira de Engenharia Agrícola e Ambiental, Campina Grande, v. 14, n. 8, p. 819-825, 2010.

PINHEIRO, E. S. Comparação entre dados altimétricos shuttle radar topography mission, cartas topográficas e GPS: numa área com relevo escarpado. Revista Brasileira de Cartografia, v. 58, n. 1, p. 1-9, 2006.

PIRES, J. M.; NASCIMENTO, M. C.; SANTANA, R. M.; RIBEIRO, C. A. A. S. Análise da exatidão de diferentes métodos de interpolação para geração de modelos digitais de elevação e obtenção de características morfométricas em bacias hidrográficas. Revista Brasileira de Recursos Hidricos, v. 10, n. 2, p. 39-47, 2005.

RABUS, B.; EINEDER, M.; ROTH, A.; BAMLER, R. The shuttle radar topography mission - a new class of digital elevation models acquired by spaceborne radar. Journal of Photogrammetry and Remote Sensing, v. 57, n. 4, p. 241-262, 2003.

REDIVO, A. L.; GUIMARÃES, R. F.; RAMOS, V. M.; CARVALHO JÚNIOR, O. A.; MARTINS, E. S. Comparação entre diferentes interpoladores na delimitação de bacias hidrográficas. Planaltina: EMBRAPA Cerrados, 2002. 20 p. (Documentos, 71).

RIBEIRO, C. A. A. S.; SOARES, V. P.; SANTOS, R. M.; SOARES, P. C. B. Estruturação topológica de grandes bases de dados de bacias hidrográficas. Revista Árvore, Viçosa, v. 32, n. 4, p. 687-696, 2008.

SANTOS, P. R. A.; GABOARDI, C.; OLIVEIRA, L. C. Avaliação da precisão vertical dos modelos SRTM para a Amazônia. Revista Brasileira de Cartografia, v. 58, n. 1, p. 101-107, 2006.

SETEGN, S. G.; SRINIVASAN, R.; DARGAHI, B.; MELESSE, A. M. Spatial delineation of soil erosion vulnerability in the Lake Tana Basin, Ethiopia. Hydrological Processes, v. 23, n. 26, p. 3738-3750, 2009. 
SEYLER, F.; MULLER, F.; COCHONNEAU, G.; GUIMARÃES, L.; GUYOT, J. L. Watershed delineation for the Amazon sub-basin system using GTOPO30 DEM and a drainage network extracted from JERS SAR images. Hydrological Processes, v. 23, n. 22, p. 31733185, 2009.

SOUZA, E. R.; FERNANDES, M. R. Sub-bacias hidrográficas: unidades básicas para o planejamento e a gestão sustentáveis das atividades rurais. Informe Agropecuário, Belo Horizonte, v. 21, n. 207, p. 15-20, 2000.
VALENTE, O. F.; DIAS, H. C. T. A bacia hidrográfica como unidade básica de produção de água. Ação Ambiental, Viçosa, v. 4, n. 20, p. 8-9, 2001.

VALERIANO, M. M.; ABDON, M. M. Aplicação de dados SRTM a estudos do Pantanal. Revista Brasileira de Cartografia, v. 59, n. 1, p. 63-71, 2007.

VALERIANO, M. M.; ROSSETTI, D. F. Topodata: Brazilian full coverage refinement of SRTM data. Applied Geography, v. 32, n. 2, p. 300-309, 2012.

WISE, S. Assessing the quality for hydrological applications of digital elevation models derived from contours. Hydrological Processes, v. 14, n. 11, p. 19091929, 2000. 
